\title{
Introduction to the Special Section on Clinical Applications of Multi-Scale Modeling
}

Throughout the history of conventional diagnostic and therapeutic processes associated with clinical science, the use of single-scale models has been quite commonplace. From basic linear system models to control physiological monitoring, drug dosage, and assistance devices to complex biophysical models to reconstruct diagnostic image content, the use of mathematical representations has become an intrinsic component of the fabric of clinical decision and treatment processes. Despite biological systems being characterized quite well with single-scale and sometimes quite sophisticated models, the ability to quantitatively represent and unify findings across geometric, functional, and temporal scales has not been as forthcoming. The goal of multi-scale modeling is to address this very challenging problem. Such an approach is predicated on the notion that accurate and precise mathematical descriptions of complex systems require characterizing features that may be invisible to overly constrained single-scale models. Furthermore, it follows that, if developed, these improved multi-scale representations will allow for new breakthroughs in our clinical understanding and treatment of disease and dysfunction. Given that intriguing premise, and that we are only now at the genesis of this new modeling initiative, it is difficult to overstate the importance of building and validating practical multi-scale models for clinical application as an important first step in this new, potentially disruptive, paradigm.

Toward this end, in 2011, the National Institutes of Health, National Science Foundation, and the Food and Drug Administration issued an announcement to establish the program in Predictive Multi-scale Models for Biomedical, Biological, Behavioral, Environmental and Clinical Research. This funding mechanism uses the U01 cooperative agreement thereby encouraging extensive data and model sharing between sites so that the productivity and progress of the consortium, as a whole, is greater than the sum of the individual contributions. A subset of the projects funded by this mechanism is focused on the clinical applications of multi-scale modeling and four of those areas are represented in this special series: surgery, drug development, oncology, and cardiology.
In the first contribution of this special section, Kassab et al. provides a multidisciplinary perspective discussing the impact of multi-scale modeling within the entirety of perioperative surgical care with examples that span an incredible breadth of applications involving surgical planning, device design, therapy guidance and delivery, and post-operative wound care. In the second paper, Eckmann et al. discuss applications of multi-scale modeling in pharmaceutical research where the range of space and time scales can stretch over several orders of magnitude thereby necessitating the methods of multi-scale modeling. The authors posit that multi-scale modeling in this field is becoming increasingly important as validated models can reduce the solution space that must be searched in the hunt for new and better drugs and delivery systems. The third paper, by Yankeelov et al., focus on the practical applications of multi-scale modeling in clinical oncology. After presenting the most common mathematical techniques employed in modeling problems of relevance to oncology and illustrative applications thereof, the authors discuss the challenges to overcome for multiscale modeling to have success in clinical oncology. The fourth and final paper, by Zhang et al., presents multi-physical models for understanding 
cardiovascular disease development and progression and how these methods can be used to design clinical interventions.

The goal of this special section is to provide a snapshot of the current state-of-the-art in multi-scale modeling as it applies to clinical problems in surgery, drug development, oncology, and cardiology. Importantly, these contributions also identify key areas that must be further investigated to realize the promise of multi-scale modeling methods. We sincerely hope the readership of Annals of Biomedical Engineering will find these four contributions engaging and informative, and that they will encourage other basic and translational scientists to continue to develop multiscale modeling techniques appropriate for clinical application.

\section{Thomas E. YANKEelov}

Departments of Biomedical Engineering and Internal Medicine, Institute for Computational and Engineering Sciences, Livestrong Cancer Institutes The University of Texas at Austin, Austin, TX, USA

Electronic mail: thomas.yankeelov@utexas.edu

\section{Michael I. Miga}

Departments of Biomedical Engineering, Neurological Surgery, Radiology and Radiological Sciences, Vanderbilt Institute in Surgery and Engineering, Vanderbilt University Medical Center, Vanderbilt University, Nashville, TN, USA 